\title{
Calculation of the vibrational linewidth and line shape of Raman spectra using the relaxation function : I. method and application to nitrogen
}

Citation for published version (APA):

Kooi, M. E., Smit, F., Michels, J. P. J., \& Schouten, J. A. (2000). Calculation of the vibrational linewidth and line shape of Raman spectra using the relaxation function : I. method and application to nitrogen. Journal of Chemical Physics, 112(3), 1395-1403. https://doi.org/10.1063/1.480693

DOI:

$10.1063 / 1.480693$

Document status and date:

Published: 01/01/2000

Document Version:

Publisher's PDF, also known as Version of Record (includes final page, issue and volume numbers)

Please check the document version of this publication:

- A submitted manuscript is the version of the article upon submission and before peer-review. There can be important differences between the submitted version and the official published version of record. People interested in the research are advised to contact the author for the final version of the publication, or visit the DOI to the publisher's website.

- The final author version and the galley proof are versions of the publication after peer review.

- The final published version features the final layout of the paper including the volume, issue and page numbers.

Link to publication

\footnotetext{
General rights

- You may freely distribute the URL identifying the publication in the public portal. follow below link for the End User Agreement:

www.tue.nl/taverne

Take down policy

If you believe that this document breaches copyright please contact us at:

openaccess@tue.nl

providing details and we will investigate your claim.
}

Copyright and moral rights for the publications made accessible in the public portal are retained by the authors and/or other copyright owners and it is a condition of accessing publications that users recognise and abide by the legal requirements associated with these rights.

- Users may download and print one copy of any publication from the public portal for the purpose of private study or research.

- You may not further distribute the material or use it for any profit-making activity or commercial gain

If the publication is distributed under the terms of Article 25fa of the Dutch Copyright Act, indicated by the "Taverne" license above, please 


\title{
Calculation of the vibrational linewidth and line shape of Raman spectra using the relaxation function. I. Method and application to nitrogen
}

\author{
M. Eline Kooi, ${ }^{\text {a) }}$ Floris Smit, Jan P. J. Michels, ${ }^{\text {b) }}$ and Jan A. Schouten \\ Van der Waals-Zeeman Institute, Valckenierstraat 65, 1018 XE Amsterdam, The Netherlands
}

(Received 7 April 1999; accepted 13 October 1999)

\begin{abstract}
The spectral line shape of the fundamental vibration of nitrogen is calculated from molecular dynamics simulations by determining the Fourier transform of the relaxation function. It has been applied to the fluid phase at various pressures and temperatures, and to solid $\delta$ - $\mathrm{N}_{2}$. The validity of the assumption that the spectrum at relatively high temperatures and pressures can be calculated by assuming that these systems are in the fast modulation regime $\left(\Delta \tau_{c} \ll 1\right)$, has been verified. A deviation of the vibrational line shape from the motional narrowing limit has been found for fluid nitrogen at low pressure, with a Kubo parameter, $\Delta \tau_{c}$, equal to 0.23 , and for the vibrational line of the molecules on the $a$ sites in $\delta$ - $\mathrm{N}_{2}$, with $\Delta \tau_{c}$ equal to 0.075. It is concluded that the value of the Kubo parameter is not an unambiguous criterion for the fast modulation regime. Moreover, a detailed comparison reveals a difference in the dynamical behavior of the molecules on the $a$ and $c$ sites. It is shown that this procedure can also be used if one does not know whether or not closely spaced lines are to be expected. The present procedure is suited to calculate line shapes in the intermediate Kubo regime, e.g., in concentrated mixtures, where no simple relations are available. Finally it is shown that in nitrogen at low density and $126 \mathrm{~K}$ the ratio of the correlation time of the frequency autocorrelation function and the dephasing time is smaller than in $\mathrm{CH}_{3} \mathrm{I}$, where the $n$ dependence of the vibrational overtone is subquadratic. (c) 2000 American Institute of Physics.
\end{abstract} [S0021-9606(00)51502-5]

\section{INTRODUCTION}

The vibrational behavior of simple fluids has been studied rather extensively during the last several decades, ${ }^{1-20}$ either by analytical methods or by computer simulations. In particular, the Raman $Q$ branch of nitrogen has been the subject of these studies. There are some obvious reasons for the choice of nitrogen for vibrational studies: the molecule is relatively simple, symmetric, nonpolar and it has only one vibrational mode. Since the rotational energy levels are very close, the rotations can be treated in several cases classically, i.e., continuously distributed at equilibrium. Moreover, the vibrational population relaxation can be considered very small, ${ }^{21}$ and therefore the linewidth is determined mainly by dephasing. Finally, the internal energy as a function of the bond length has been determined quantitatively. ${ }^{22}$

A thorough treatment of the theory of line shapes is given by Kubo. ${ }^{1}$ A concise overview of the essentials of this theory will be given in the following section. Oxtoby and co-workers ${ }^{6-8}$ have studied in detail the various contributions to the Raman line shift and the dephasing time of liquid nitrogen at its boiling point by computer simulations. They did not determine the relaxation function for calculating the dephasing time but obtained it from the frequency autocorrelation function. In a very recent article Gayathri and Bagchi $^{23}$ reported computer simulations to investigate the

\footnotetext{
a) Present address: Academic Hospital Maastricht, University of Maastricht, Department of Radiology, P. Debeyelaan 25, 6229 HX Maastricht, The Netherlands.

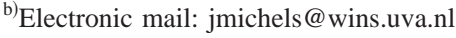

subquadratic quantum number dependence of vibrational overtone dephasing. They calculated the relaxation function of liquid $\mathrm{N}_{2}$ and liquid $\mathrm{CH}_{3} \mathrm{I}$ and found that the nonquadratic dependence can be expected when the decay of the frequency autocorrelation function is largely Gaussian and the time scale of this decay is comparable to that of the autocorrelation function of the normal coordinate.

Our work is primarily focused on the calculation of the Raman linewidth and line shape, which requires Fourier analyses of the relaxation function. In their simulations Gayathri and Bagchi (GB) did not take into account the dispersion correction (DC). In a previous paper $^{17}$ we have shown that the DC gives a considerable contribution to the linewidth (and thus to the dephasing time). Vibrationrotation (VR) coupling is also important, in particular at higher temperatures. GB neglected the cross correlation between the VR term and the other contributions. However, these cross terms have a larger value and are of opposite sign compared to that of the autocorrelation. ${ }^{8,20}$ This also explains the discrepancy between the results of Levesque, Weis, and Oxtoby $^{8}$ and of GB with respect to the VR term. Finally, as stated in Ref. 8: “...one needs to know the potential surface very accurately in order to predict quantitatively the vibrational line shape." Therefore, we have used the van der Waals part of the Etters potential. ${ }^{24}$ To speed up the calculations we have omitted the quadrupolar interaction.

In both the fast and the slow modulation regime the linewidth can easily be calculated ${ }^{1}$ from the amplitude of modulation $\Delta$ and the decay or correlation time of the frequency $\tau_{c}$, while in the intermediate regime a Fourier analysis of the 
relaxation function is needed. The first calculation of the linewidth, using the relaxation function, has been carried out by Levesque, Weis, and Oxtoby ${ }^{7}$ for liquid $\mathrm{HCl} \cdot \mathrm{HCl}$ is an intermediate case. Quantitative agreement was not obtained, probably due to an inaccurate potential surface. In the case of nitrogen a very good site-site interaction potential is available $^{24}$ and better quantitative agreement is anticipated. In this work a different procedure has been applied for reasons to be explained.

The condition for the fast modulation regime is ${ }^{1}$ given by $\Delta \tau_{c} \ll 1$, thus it is not clear when the transition to the intermediate regime starts. In binary mixtures with high concentration of both components concentration fluctuations also play a role. These are considerably slower than density fluctuations and the system may then be in the intermediate Kubo regime. In this paper we will test the procedure to be used in the Fourier analysis by applying it in the fast modulation regime, where a comparison can be made with the Kubo expressions. The method will be applied to nitrogen at different densities and temperatures in order to get information about the range of the fast modulation regime and about relative changes in the various contributions in going from the fast to the intermediate regime. It might also give some insight into the effect of the time scales as discussed by Gayathri and Bagchi. ${ }^{23}$ In paper II we will apply the method to the calculation of inhomogeneously broadened line shapes in mixtures of neon and nitrogen at high pressures and compare the results with experimental data.

\section{THEORY}

A stochastic theory of line shape and relaxation is given by Kubo. ${ }^{1}$ This theory considers an oscillator with a frequency $\omega_{i}$, which is randomly modulated. For ease of understanding the essential equations will be briefly given below. In the theory the following properties are defined

(a) The probability density $P(\omega)$ which represents the continuous distribution of the momentary frequencies over an extended range.

(b) The amplitude of modulation, defined as the standard deviation of $P(\omega)$ :

$$
\Delta=\left\{\left\langle\omega^{2}\right\rangle-\langle\omega\rangle^{2}\right\}^{1 / 2} .
$$

(c) The frequency autocorrelation function:

$$
\Omega(t)=\frac{\langle\omega(0) \omega(t)\rangle-\langle\omega(0)\rangle^{2}}{\Delta^{2}} .
$$

(d) The correlation time:

$$
\tau_{c}=\lim _{t \rightarrow \infty} \int_{0}^{t} \Omega(s) d s .
$$

(e) The relaxation function:

$$
\varphi(t)=\left\langle\exp \left\{i \int_{0}^{t} d t^{\prime} \omega\left(t^{\prime}\right)\right\}\right\rangle .
$$

For two limiting cases the line shape is given by the following simple expressions (a) The so-called fast modulation regime for $\Delta \tau_{c} \ll 1$. In this regime the line shape is Lorentzian and the full width at half-maximum (FWHM) is given by $2 \Delta^{2} \tau_{c}$.

(b) The so-called slow modulation regime for $\Delta \tau_{c} \gg 1$. In this regime it turns out that the line shape reflects the momentary frequency distribution, $P(\omega)$, and the FWHM is given by $2 \Delta$.

According to the fluctuation dissipation theory the line shape is given by the Fourier transform of the relaxation function:

$$
I(\omega)=\frac{1}{2 \pi} \int_{-\infty}^{\infty} e^{-i \omega t} \phi(t) d t .
$$

Kubo has shown that for any stochastic process with $\omega(t)=\omega_{0}+\omega_{1}(t)$ the relaxation function can be expanded as

$$
\phi(t)=\left\langle\exp \left\{i \int_{0}^{t} \omega\left(t^{\prime}\right) d t^{\prime}\right\rangle=e^{i \omega_{0} t} \exp \{K(t)\}\right.
$$

with

$$
K(t)=-\frac{1}{2} \int_{0}^{t} \int_{0}^{t}\left\langle\omega_{1}\left(t_{1}\right) \omega_{1}\left(t_{2}\right)\right\rangle d t_{1} d t_{2}+\cdots .
$$

For $t \gg \tau_{c}, K(t)$ is given by the following asymptotic form:

$$
K(t)=-t\left\{\Delta^{2} \tau_{c}+O\left(\Delta^{3} \tau_{c}^{2}\right)+\cdots+O\left(\Delta^{n+1} \tau_{c}^{n}\right)+\cdots\right\} .
$$

Therefore, for any stochastic process with $\omega(t)=\omega_{0}$ $+\omega_{1}(t)$, the amplitude of the relaxation function decays exponentially for $t \gg \tau_{c}$.

In the fast modulation regime where $\Delta \tau_{c} \ll 1$, the following equation for $K(t)$ can be deduced:

$$
K(t)=-t\left\{\Delta^{2} \tau_{c}+\Delta \sum_{n=2}^{\infty} O\left(\Delta \tau_{c}\right)^{n}\right\} \rightarrow-\Delta^{2} \tau_{c} t .
$$

Thus, the absolute value of the relaxation function in this regime is given by

$$
\begin{aligned}
& |\phi(t)|=e^{-\Delta^{2} \tau_{c} t}=e^{t / T_{2}}, \\
& \text { with } T_{2}^{-1}=\Delta^{2} \tau_{c} .
\end{aligned}
$$

This results in a Lorentzian shape of the spectral line in the case of strong motional narrowing, with a FWHM equal to $2 \Delta^{2} \tau_{c}$.

\section{SIMULATION METHOD}

The procedure applied for the calculation of the vibration frequency from molecular dynamical calculations has been described extensively in earlier papers, ${ }^{17,19,20,25,26}$ but for the convenience of the reader, we recapitulate the most essential topics below.

For the model particles of nitrogen a site-site potential has been adopted, in which each site corresponds to an atom site. The distance between two atoms of the same molecule is $1.094 \AA$. The interaction potential between atoms of distinct molecules was taken from the Etters ${ }^{24}$ potential $V(r)$ :

$$
V(r)=A_{1} \exp \left(-\alpha_{1} r\right)-B_{1} r^{-6}, \quad r>R_{1},
$$


TABLE I. Calculation results of nitrogen in the fluid phase. Note that the frequencies $\nu_{1}$ are given with respect to the frequency of an isolated nonrotating molecule.

\begin{tabular}{lcccc}
\hline \hline \multicolumn{1}{c}{ System } & $\mathrm{a}$ & $\mathrm{b}$ & $\mathrm{c}$ & $\mathrm{d}$ \\
\hline$T(\mathrm{~K})$ & 126 & 126 & 296 & 408 \\
$\rho\left(\mathrm{kmol} / \mathrm{m}^{3}\right)$ & 9 & 30 & 47 & 52.5 \\
$p(\mathrm{GPa})$ & 0.004 & 0.114 & 2.4 & 4.37 \\
$\left\langle\nu_{1}\right\rangle\left(\mathrm{cm}^{-1}\right)$ & -2.018 & -3.926 & 3.692 & 11.434 \\
$\Delta^{(1 / \lambda)}\left(\mathrm{cm}^{-1}\right)$ & 1.18 & 1.83 & 6.66 & 9.91 \\
$\nu_{1, \text { peak }}$ of $P\left(\nu_{1}\right)\left(\mathrm{cm}^{-1}\right)$ & -1.75 & -4.50 & 1.25 & 7.80 \\
Momental skew & -0.07 & 0.94 & 0.84 & 0.82 \\
Pearson's skew & -1.77 & 0.31 & 0.37 & 0.37 \\
$\tau_{c}(\mathrm{ps})$ & $1.04 \pm 0.07$ & $0.0758 \pm 0.0015$ & $0.0736 \pm 0.0015$ & $0.0656 \pm 0.0013$ \\
$\Delta \tau_{c}$ & 0.23 & 0.03 & 0.09 & 0.12 \\
$2 \Delta^{2} \tau_{c}$ & $0.55 \pm 0.04$ & $0.095 \pm 0.002$ & $1.23 \pm 0.02$ & $2.43 \pm 0.05$ \\
$\nu_{1, \text { spectrum }}\left(\mathrm{cm}^{-1}\right)$ & -1.96 & -3.93 & 3.66 & 11.39 \\
$\mathrm{FWHM}_{\text {spectrum }}\left(\mathrm{cm}^{-1}\right)$ & $0.50 \pm 0.01$ & $0.095 \pm 0.002$ & $1.20 \pm 0.01$ & $2.43 \pm 0.01$ \\
$\chi^{2}(\mathrm{Gauss}) / \chi^{2}\left(\right.$ Lorentz $\left.^{2}\right)$ & 14 & $2 \times 10^{4}$ & $1 \times 10^{3}$ & $2 \times 10^{2}$ \\
$T_{2}^{-1}\left(10^{9} \mathrm{~s}^{-1}\right)$ & $49.3 \pm 6.0$ & $8.8 \pm 0.4$ & $114.6 \pm 6.6$ & $231 \pm 15$ \\
$\left(\pi c T_{2}\right)^{-1}\left(\mathrm{~cm}^{-1}\right)$ & $0.52 \pm 0.06$ & $0.093 \pm 0.004$ & $1.22 \pm 0.07$ & $2.45 \pm 0.07$ \\
$t_{n}(\mathrm{ps})$ & 20 & 2 & 1 & 2 \\
$t_{m}(\mathrm{ps})$ & 35 & 100 & 15 & 10 \\
\hline \hline
\end{tabular}

$$
\begin{aligned}
& V(r)=\sum_{i=0}^{4} C_{i}\left(r-R_{0}\right)^{n}-B_{1} r^{-6}, \quad R_{1}>r \geqslant R_{0}, \\
& V(r)=A_{2} \exp \left(-\alpha_{2} r\right)-B_{1} r^{-6}, \quad r<R_{0}
\end{aligned}
$$

with

$$
\begin{array}{ll}
A_{1}=9.261205 \times 10^{7} \mathrm{~K}, & C_{1}=-1446.74414 \mathrm{~K}^{-1}, \\
A_{2}=1.47248 \times 10^{7} \mathrm{~K}, & C_{2}=2480.73711 \mathrm{~K}^{-2}, \\
B_{1}=1.79 \times 10^{5} \mathrm{~K}^{6}, & C_{3}=-2766.5419 \mathrm{~K}^{-3}, \\
R_{0}=3.01006875 \AA, & C_{4}=1574.2809 \mathrm{~K}^{-4}, \\
R_{1}=3.4494569 \AA, & \alpha_{1}=4.037 \AA^{-1}, \\
C_{0}=415.73107 \mathrm{~K}, & \alpha_{2}=3.48 \AA^{-1},
\end{array}
$$

but the quadrupolar term has been omitted.

The molecular dynamics (MD) simulations have been performed on a system consisting of 384 model particles in a box with periodic boundaries. During the simulation, four terms that contribute to a change of the frequency with respect to a nonrotating, isolated molecule were registered:

(a) The external force acting along the molecular axis (E1).

(b) The derivative of the external force along the axis, contributing to the harmonic force of the vibrator (E2).

(c) The vibration-rotation coupling (VR).

(d) The dispersion correction, which corrects for the change in polarizability at excitation of the vibrator (DC).

Numerical values for the conversion of forces and energy to frequency are given in Ref. 17. The parameters for the DC given in Ref. 17 are obtained at ambient temperature only. This may lead to some error in the results of the simulations of low temperature systems, but this is not relevant for the purpose of this study. Registrations were made in equidistant time steps, typically corresponding to $0.01 \mathrm{ps,} \mathrm{and} \mathrm{through-}$ out a simulated time of $\approx 1000$ ps. The integration of the equations of motion was performed in steps of $\frac{1}{6} \times 0.01 \mathrm{ps}$. On line with the simulation calculations, the mean frequency, the standard deviation or amplitude of modulation $\Delta$ (1), and the frequency autocorrelation function $\Omega(t)$ (2) have been determined. The latter function was determined throughout 300 register time steps. Next, the correlation time $\tau_{c}$ (3) was calculated by numerical integration of $\Omega(t)$. Moreover, in contrast with the previous MD calculations, the momentary frequency of all particles at each time step was put on file for further analysis.

\section{ANALYSIS AND RESULTS}

Four systems of fluid nitrogen at different densities and/or temperatures have been simulated. The determination of the linewidth has been performed by the full analysis of the relaxation function and, for comparison, by assuming that the systems are in the fast modulation regime, and thus, that the line shape is Lorentzian, as has been done in the previous work. The numerical results are given in Table I (systems a-d). The first rows display the static properties. Temperatures and densities are chosen in the supercritical range; at the two highest densities and pressures the system is just below the melting point. Next, the amplitude of modulation and the mean frequency change, $\nu_{1}$, with respect to the frequency of a nonrotating, isolated molecule $\left(\nu_{0}\right.$ $\left.=2329.91 \mathrm{~cm}^{-1}\right)^{22}$ are given. In conformity with the common usage in experimental spectroscopy, the frequencies, $\nu_{1}$, as well as the amplitudes, $\Delta^{(1 / \lambda)}$, are given in wave numbers $(\nu=\omega / 2 \pi c)$. In Fig. 1 the distribution $P\left(\nu_{1}\right)$ of the momentary frequencies is pictured: The differences among the four systems are evident. Not only the widths differ, but also the peak positions. As noted before, the shift of the momentary frequency is composed of four contributions: namely the first- (E1) and second-order (E2) effect of the external forces, the VR coupling, and the dispersion correc- 


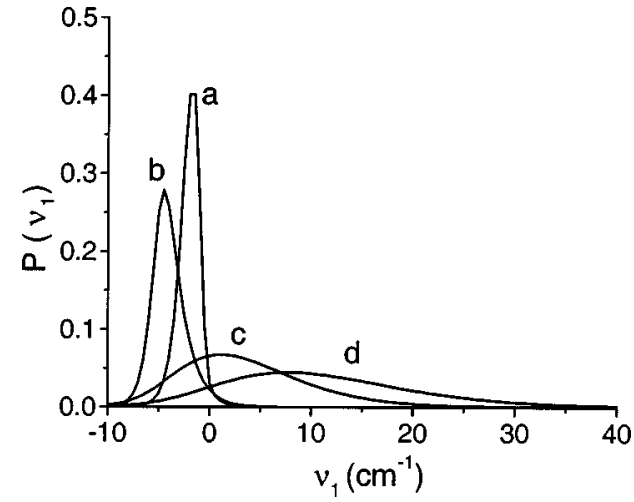

FIG. 1. Momentary frequency distribution functions of nitrogen in the fluid phase; a-126 K and $0.004 \mathrm{GPa}$; b-126 K and $0.114 \mathrm{GPa}$; $\mathrm{c}-296 \mathrm{~K}$ and $2.4 \mathrm{GPa}$; $-408 \mathrm{~K}$ and $4.37 \mathrm{GPa}$

tion (DC). The first two terms are positive in the systems considered; the third is always negative and the last is, at least in these examples, also negative. The mean frequency change $\left\langle\nu_{1}\right\rangle$ and the peak positions are negative for the first two examples, but positive for the other systems. A close look at $P\left(\nu_{1}\right)$ reveals a skewness, which can also be deduced from the difference between the numbers for $\left\langle\nu_{1}\right\rangle$ and the peak position of $P\left(\nu_{1}\right)$ in Table I. There are several definitions for the skewness, e.g., the momental and the Pearson's skewness. Numbers for it are given in Table I. The importance of the absolute values will not be discussed, but the sign of the skewness numbers must be noted.

The behavior of the frequency self-correlation functions $\Omega(t)$ for the sample systems during the first picosecond are displayed in Fig. 2. In all cases, $\Omega(t)$ decreases rapidly during the first moments, whereupon a modulation followed by a slow decrease is seen. This long time behavior is most pronounced at the lowest density (a). This behavior is primarily caused by the long persistence of rotations and thus by the VR coupling.

The correlation time $\tau_{c}$ has been obtained by numerical integration of $\Omega(t)$ (2). For the three highest densities considered $(b, c, d)$, this function is virtually zero after 3 ps, (i.e., the length of the determination of $\Omega(t)$ but not for system a. Yet, we made an estimation of the contribution for $t>3 \mathrm{ps}$ by fitting an exponential function above $1.1 \mathrm{ps}$ and used this

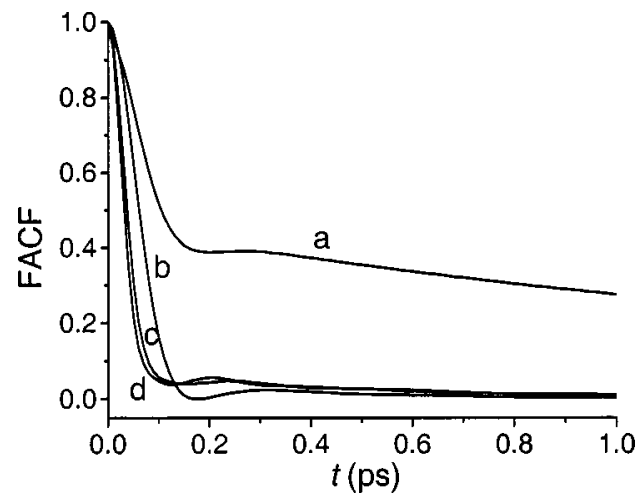

FIG. 2. Frequency autocorrelation functions of nitrogen in the fluid phase; $\mathrm{a}-126 \mathrm{~K}$ and $0.004 \mathrm{GPa}$; $-126 \mathrm{~K}$ and $0.114 \mathrm{GPa}$; $-296 \mathrm{~K}$ and $2.4 \mathrm{GPa}$; $\mathrm{d}-408 \mathrm{~K}$ and $4.37 \mathrm{GPa}$. for extrapolation from $t=3$ ps to $t \rightarrow \infty$. The results for the correlation times are given in Table I. The accuracy for b, c, and $\mathrm{d}$ is about $2 \%$, but for a, with an extrapolation contributing nearly $30 \%$ of the total result, the uncertainty is hard to determine. We estimate that the total uncertainty in $\tau_{c}$ is about $7 \%$, providing that the decay is exponential. The next row in Table I denotes the values for $\Delta \tau_{c}$. As mentioned in the previous section, the Kubo theory predicts a Lorentzian line shape with a width (FWHM) of $2 \Delta^{2} \tau_{c}$ if $\Delta \tau_{c} \ll 1$. It is clear that this condition is most questionable for a, and best realized for $\mathrm{b}$. The numbers for the width of the assumed Lorentzian lines are given in the next row of the table.

For the high temperature cases $\mathrm{c}$ and $\mathrm{d}$ a detailed discussion of the contribution of the various mechanisms and cross correlations to the linewidth has been given in Ref. 20. It has been shown that the contribution of the dispersion correction to the linewidth depends on the pressure and amounts to more than $20 \%$ at the highest pressures. The cross correlations are also important, in particular those with the VR coupling which give a negative contribution to the correlation time of the frequency. Because of the low density, in case a all the correlation times increase considerably compared to cases $\mathrm{c}$ and $\mathrm{d}$, in particular that of the VR coupling and to a smaller degree that of the DC. On the other hand the amplitudes of modulation are much smaller, although the relative contribution of VR has increased. In case b the correlation times are comparable to $c$ and d, but that of the DC is longer than at room temperature at about the same density $(0.45$ $\mathrm{GPa}$ in Ref. 20) and the contribution of VR is less pronounced. The amplitudes of modulation are smaller than at room temperature.

The above-described method has shown to be rather successful in the previous work. A fair correspondence with experimental results for the linewidth was found for the systems considered. ${ }^{17,19,25,26}$ Nevertheless, it is obvious that the a priori assumption of fast modulation and thus, of a Lorentzian line shape, must be regarded as a serious restriction of the applicability. As is well known, it would be preferable to calculate the line directly from the dephasing by the Fourier transform of the relaxation function, ${ }^{7}$ according to Eq. (5). In principle, this can be done straightforwardly, but the results for $\phi(t)$ turn out to be rather inaccurate due to the restricted number of particles, as is demonstrated in Fig. 3(a). Consequently, the Fourier transform is scattered as seen in Fig. $3(b)$, so that the line shape cannot be determined.

In order to improve the statistics we made use of the fact that for $t \gg \tau_{c}, \Omega(t) \rightarrow 0$, and, according to Eq. (7), the relaxation function decays exponentially. The MD simulation provides us with a matrix of frequencies $\nu_{1 i j}$ with $K \times L$ elements, where $K$ is the number of model particles, and $L$ the number of time steps. For each particle the relaxation function has been calculated by numerical integration for $0<t$ $<t_{m}$. At $t_{n} \gg \tau_{c}$, with $t_{n}<t_{m}$, this procedure is repeated, as if starting at $t=0$ again. The procedure is redone at $t_{2 n}$, etc., until the last possible full series of $m$ time steps. The average of all these series provides us with the relaxation function, $\phi(t)$ for $0<t<t_{m}(4)$. By these means, the statistics is increased by an order of magnitude. An exponential function is fitted for $\phi(t)$ in the range $t_{n}<t<t_{m}$ by the least-squares 


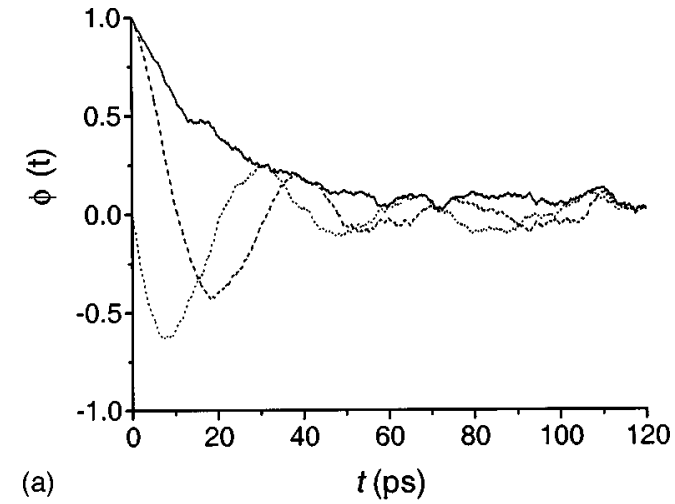

(a)

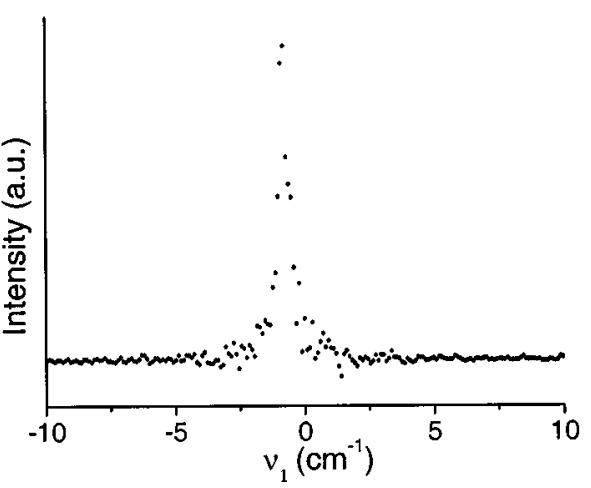

FIG. 3. (a) Relaxation function obtained directly from the simulation with the restricted statistics at $296 \mathrm{~K}$ and $1.2 \mathrm{GPa}$ with 256 particles; dashed line, real part; dotted line, imaginary part; solid line, absolute value. (b) Calculated spectrum: Fourier transformation of the relaxation function given in (a).

method. For $t>t_{m}$, the relaxation function has been extrapolated using the exponential fit. In Ref. 7 the initial part of $\phi(t)$ has been fitted with a Gaussian but in this work we used the original data. The spectral lines have been obtained by calculating the Fourier transformation of $\phi(t)$. The peak position $I\left(\nu_{\max }\right)$ is determined by fitting a parabola through a few points around the peak position. In order to determine the FWHM, two parabolas have been fitted using the linear least-squares method through the two sets of points at both sides of the peak position with about half the maximal intensity. These parabolas have been used for interpolation in order to obtain the FWHM.

The relaxation functions of the four systems in the fluid phase have been plotted on a logarithmic scale in Figs. 4(a) and 4(b). For $t \gg \tau_{c}$ these functions indeed decay exponentially. The relaxation functions of systems $b-d$ already decay exponentially for $t>0.2 \mathrm{ps}$ as shown in Fig. 4(b), and for system a it sets in at $t \approx 2 \mathrm{ps}$. Therefore $t_{n}$ should be chosen larger than 0.2 ps for systems $b-d$, and larger than 2 ps for system a. In addition, $t_{n}$ must be chosen much larger than $\tau_{c}$. These conditions are fulfilled (Table I). It has been verified that the calculated spectral line is not sensitive to the choice of the value of $t_{n}$. The accuracy of $\phi(t)$ is determined by the total contribution of the extrapolation and the error in the exponential fit. Therefore, the accuracy increases for larger values of $t_{m}$. However, the value of $t_{m}$ must not be chosen too large in order to obtain smooth spectra. This is attributed to the fact that the value of $\phi(t)$ is very small for large $t$, which leads to numerical errors. Indeed for the sys-
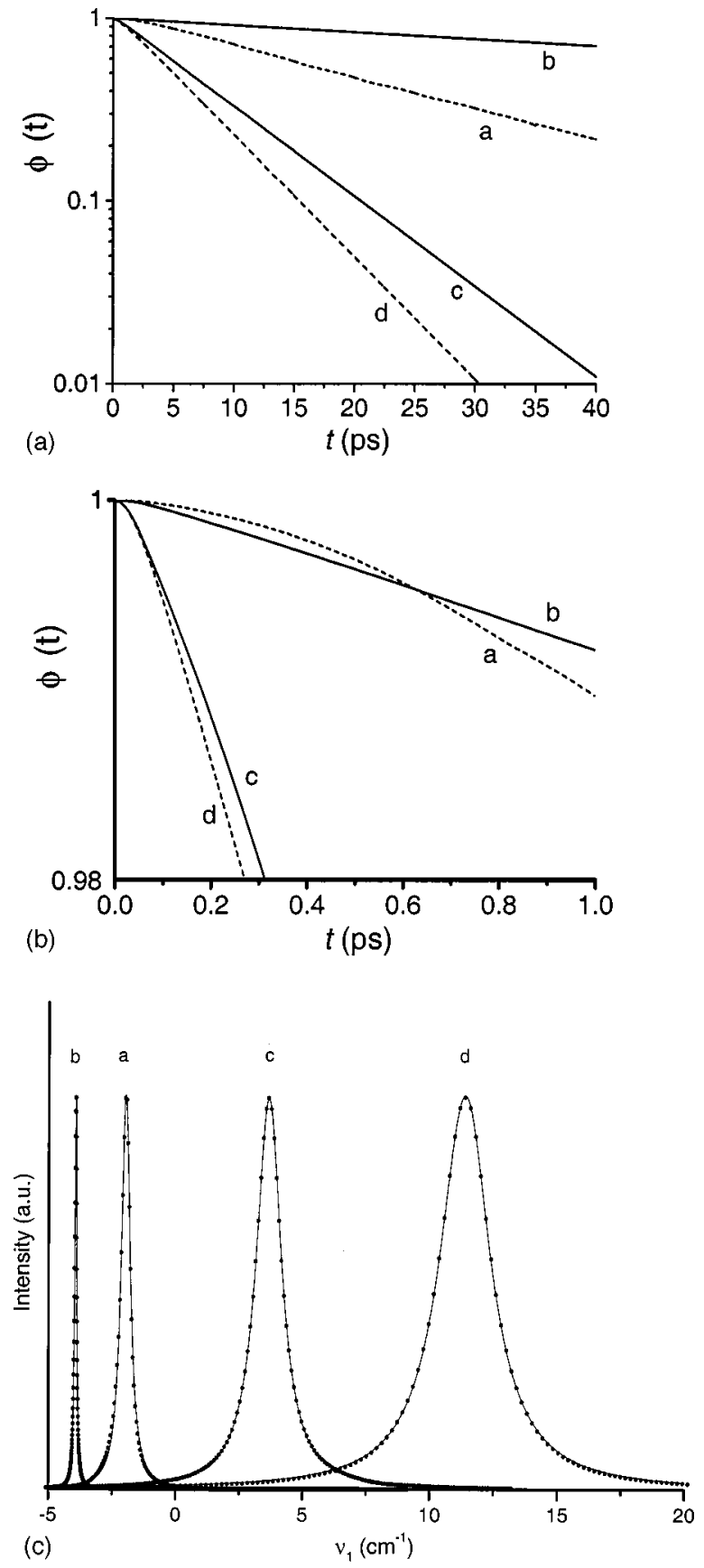

FIG. 4. (a) Absolute values of relaxation functions plotted on a logarithmic scale of the four systems in the fluid phase from $t=0-40 \mathrm{ps}$. The results have been obtained by using the following values for $t_{n}$ and $t_{m}$ in picoseconds. System $\mathrm{a}-t_{n}=20, t_{m}=35$; system $\mathrm{b}-t_{n}=2, t_{m}=100$; system $\mathrm{c}-t_{n}$ $=1, t_{m}=15$; system $\mathrm{d}-t_{n}=2, t_{m}=10$. (b) Absolute values of relaxation functions plotted on a logarithmic scale of the four systems in the fluid phase from $t=0$ to 1 ps. (c) Calculated spectra (circles) of the four systems. The spectra have been obtained by Fourier transformation of the relaxation functions given in (a). The lines represent Lorentzian fits of the calculated spectra. Note that the spectra have not been normalized, and that the frequencies $\nu_{1}$ are given with respect to the frequency of an isolated nonrotating molecule.

tems with a fast decay of the relaxation function ( $c$ and d), this effect occurs already for smaller values of $t_{m}$, than for the systems with a slower decay ( $a$ and b). As long as the condition $\phi\left(t_{m}\right)>0.05$ is fulfilled, smooth spectra have been obtained. If the value of $t_{m}$ is chosen according to the above- 
given conditions, the final results do not depend on the choice of $t_{m}$. The Raman spectrum of system $\mathrm{c}$ has been simulated by using only 12000 time steps (one time step, $d t=0.01 \mathrm{ps}$ ), as well as by using 50000 time steps. Within the accuracy of the calculations the calculated spectra are the same. For all the calculations described in this work at least 50000 time steps have been used. The influence of the choice of the time interval between steps, $d t$, has been investigated by performing three MD simulations on system d, with the smallest correlation time of the systems considered in this work, with time intervals, $d t$, of $0.01,0.005$, and $0.0025 \mathrm{ps}$, respectively. Within the accuracy of the calculations these three simulations resulted in the same calculated spectrum.

The four systems of nitrogen in the fluid phase have been analyzed by calculating the Fourier transform of the relaxation function (5). It is shown that system $d$ exhibits the fastest decay of the relaxation function. The decay of the relaxation function of system $b$ is very slow, even at $200 \mathrm{ps}$ the value of $\phi(t)$ is still larger than 0.17. Thus, for this system the dephasing time is very long, resulting in a narrow line. The exponents, $T_{2}^{-1}$, of the exponential fits of the absolute value of the relaxation functions in the range $t_{n} \leqslant t$ $\leqslant t_{m}$,

$$
|\phi(t)|=\alpha \exp \left\{\frac{-t}{T_{2}}\right\}
$$

have been plotted in Table I. Considering the error in the exponential fit and the total contribution of the extrapolation, the relaxation function is determined with an accuracy of $2.2 \%, 1.9 \%, 1.0 \%$, and $0.6 \%$ for systems a, b, c, and $\mathrm{d}$, respectively. The calculated spectra are presented in Fig. 4(c). The points represent the calculated spectra, and the lines are Lorentzian fits of these spectra. It can be noticed that the fits are good for the spectra of the systems b, c, and $\mathrm{d}$, although the spectra $\mathrm{c}$ and $\mathrm{d}$ are slightly asymmetric. The line shape of the calculated spectrum a deviates from a Lorentzian curve. This can be observed in Fig. 4(c) since the points in the wing of spectrum a, at the low frequency side of the peak position, have a higher intensity than the Lorentzian curve. Note, that the value of $\Delta \tau_{c}$ is the largest for system a. The asymmetries of the spectra a, c, and $\mathrm{d}$ are reflected in the peak positions of the calculated spectra, given in Table I, that deviate slightly from the average frequency of the momentary distribution function. The value of the peak position of the calculated spectrum of system $b$, with the smallest value of $\Delta \tau_{c}$, is equal to the average frequency of the momentary distribution function. The FWHMs of the calculated spectra are much smaller than twice the standard deviation of the momentary frequency distribution. That the effect of motional narrowing can be very large is demonstrated clearly by system b. In this case, the FWHM of the calculated spectrum is about 40 times less than that of the momentary frequency distribution. The FWHMs of the spectra a, c, and d deviate from the values of $2 \Delta^{2} \tau_{c}$ by $-12 \%,-1.6 \%$, and $+1.2 \%$, respectively. The calculated spectrum of system b shows perfect agreement with the Kubo prediction of the spectral line shape in the motional narrowing regime: a Lorentzian line shape with a FWHM equal to $2 \Delta^{2} \tau_{c}$. It is interesting to note that in time-resolved spectroscopy the dephasing time is determined. In the motional narrowing regime $\left(\Delta \tau_{c} \ll 1\right)$ the FWHM in $\mathrm{cm}^{-1}$ of the spectral line is equal to $\left(\pi c T_{2}\right)^{-1}$ (9). In our calculations $T_{2}^{-1}$ corresponds to the exponent of the absolute value of the relaxation function. Therefore, these values are given in Table I. It is shown that these values are the same as the FWHM of the calculated spectra, within the accuracy of $T_{2}^{-1}$.

An indication for a good correspondence between the calculated spectrum and the fit is given by a small value of $\chi^{2}$ defined as

$$
\chi^{2}=\sum_{i} \frac{y_{i}^{\text {spectrum }}-y_{i}^{\text {fit }}}{\sigma_{i}^{2}},
$$

where $y_{i}^{\text {spectrum }}$ is the calculated spectrum, $y_{i}^{\text {fit }}$ the fit through the calculated spectrum, and $\sigma_{i}$ the standard deviation. In Table I the ratio of $\chi^{2}$ of a Gaussian and a Lorentzian fit of the calculated spectra are given. For all four systems these ratios are much larger than unity, indicating that the calculated spectra can be fitted much better with a Lorentzian than a Gaussian curve.

Gayathri and Bagchi ${ }^{23}$ suggest that the subquadratic $n$ dependence of the overtone dephasing time as found in $\mathrm{CH}_{3} \mathrm{I},{ }^{14}$ which is in contradiction to the quadratic $n$ dependence of the Kubo-Oxtoby theory of line shapes, can be expected when the decay of the frequency autocorrelation function is largely Gaussian and the time scale of this decay is comparable with that of the decay of the relaxation function. This could happen if (a) the frequency is not too large, (b) the amplitude of modulation is large, and (c) the anharmonicity is large. These conditions are not satisfied in the case of nitrogen. ${ }^{23}$ However, in case a the deviation of the line shape from a Lorentzian is very clear and the ratio of the dephasing time and correlation time is about 20, as compared to about 40 for $\mathrm{CH}_{3} \mathrm{I}$. Therefore, it would be interesting to test the suggestions of Gayathri and Bagchi by measuring the $n$ dependence of the vibrational overtones of nitrogen under the conditions of case a. For case $d$ the ratio is also small (about 65) but the peak shows only a slight asymmetry.

Finally, the applicability of the method will be demonstrated by calculating the vibrational spectrum of solid nitrogen in the $\delta$ phase. Quadrupolar interactions are important for the structure of the solid phase. We have performed a few calculations, using the frequency modulation time correlation function, which show that in $\delta$ nitrogen also the linewidth depends on the quadrupolar forces but by far the largest contribution comes from the van der Waals part of the potential. The effect on the shift of the frequency is relatively small. Moreover, the quadrupolar forces have a long range so that a large number of particles is needed in the simulations. In order to save computer time, the majority of the calculations were performed while omitting the quadrupolar forces.

The cubic unit cell of $\delta$ - $\mathrm{N}_{2}(P m 3 n)$ contains eight molecules. ${ }^{27-29}$ The two molecules on the $a$ sites are completely orientational disordered and the six molecules on the $c$ sites have a random orientation in a plane normal to the faces of the unit cell. Therefore, the Raman spectrum consists of two distinct peaks, corresponding to the frequencies of the molecules on the $a$ and $c$ sites, respectively. Ideally, 

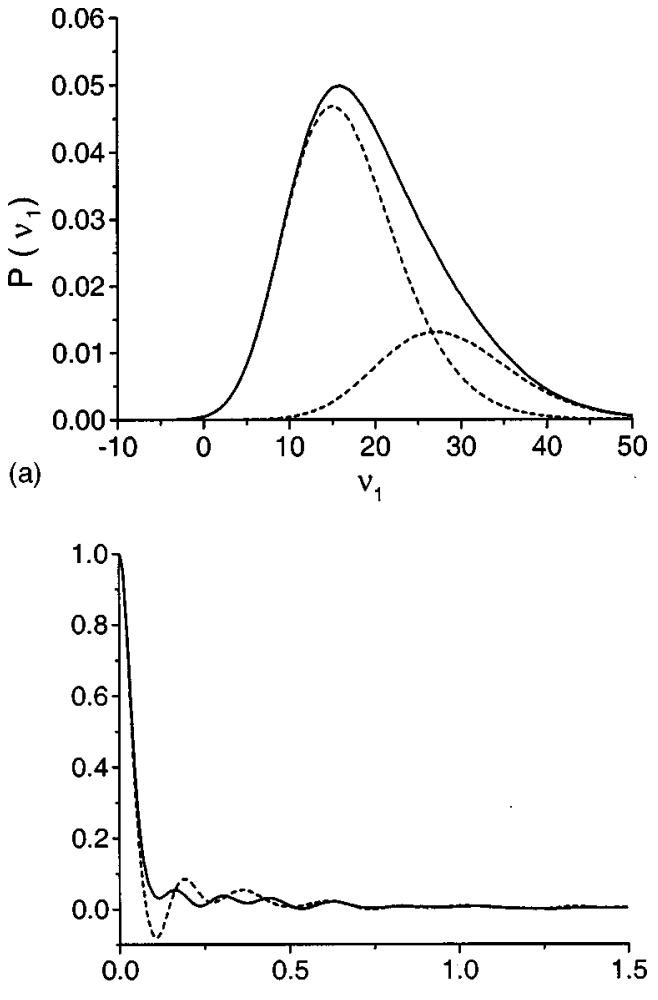

(b)

$t$ (ps)

FIG. 5. (a) Momentary frequency distribution function of the Raman frequencies of nitrogen in the $\delta$ phase at $170 \mathrm{~K}$ and $7.0 \mathrm{GPa}$. The dashed lines represent the momentary distribution functions of the molecules on the $a$ sites (high frequency distribution) and $c$ sites (low frequency distribution), respectively. The solid line represents the total momentary distribution function. (b) Frequency autocorrelation functions of the Raman frequencies corresponding to the $a$ sites (dashed line) and to the $c$ sites (solid line) of nitrogen in the solid $\delta$ phase at $170 \mathrm{~K}$ and $7.0 \mathrm{GPa}$.

the calculation of these two peaks should be performed by analyzing the frequencies of the two sites separately, since for $t \gg \tau_{c}$ the relaxation functions of the frequencies corresponding to the two different sites decay with a different exponent. In order to show the power of the method we will perform the calculations also by considering it as one single relaxation function. The momentary distribution functions, $P\left(\nu_{1}\right)$, have been plotted in Fig. 5(a). The dashed lines represent the functions of the molecules on the $a$ and the $c$ sites, respectively, and the solid line represents the total function. The autocorrelation functions have been plotted in Fig. 5(b). It is shown that this function for the molecules on the $a$ sites falls off more rapidly than that of the molecules on the $c$ sites, and even becomes negative. A calculation of the correlation functions of the various mechanisms shows that the more rapid decay in the case of the $a$ sites is primarily due to the first-order effect (E1). The autocorrelation function (E1E1) of the $a$ sites shows a negative part after a time of about $0.1 \mathrm{ps}$. The other autocorrelation functions are always positive. All the cross-correlation functions of the $a$ sites have a small negative part. The negative contributions of E1-VR, E1-DC, and E1-E2 are the most important although the value is less than that of E1-E1. Of the $c$ sites only the cross correlations with VR give a negative contribution. The most important is E1-VR. The amplitude of modulation is smaller for the $c$ sites than for the $a$ sites (Table II). The relative contribution of most of the mechanisms to the value of $\Delta$ is nearly the same for both sites, but the relative contribution of $\mathrm{E} 1-\mathrm{E} 1$ is $10 \%$ less for the $a$ sites and that of E1-DC is $20 \%$ more.

First, the vibrational spectra have been calculated by analyzing the frequencies of the two sites separately. The results of the calculations are presented in Table II. The relaxation function of the vibrational frequency of the molecules on the $a$ sites decays much faster than that of the molecules on the $c$ sites. For both relaxation functions the exponential decay sets in for $t \approx 0.7 \mathrm{ps}$. The calculated spectra show good correspondence with Lorentzian curves. The spectral line shape of the peak corresponding to the $c$ sites shows perfect agreement with the Kubo predictions of a spectral line shape in the fast modulation regime. The spec-

TABLE II. Calculation results of nitrogen at $170 \mathrm{~K}$ and $7.00 \mathrm{GPa}$ and $60.96 \mathrm{kmol} / \mathrm{m}^{3}$ in the $\delta$ phase. Note that the frequencies $\nu_{1}$ are given with respect to the frequency of an isolated nonrotating molecule.

\begin{tabular}{|c|c|c|c|c|c|}
\hline & \multicolumn{2}{|c|}{$\begin{array}{c}\text { Separate calculation of } \\
\text { relaxation function of } a \text { and } c \\
\text { sites }\end{array}$} & \multicolumn{3}{|c|}{$\begin{array}{c}\text { Calculation without } \\
\text { distinguishing between } a \\
\text { and } c \text { sites }\end{array}$} \\
\hline & $a$ sites & $c$ sites & $a$ sites & & $c$ sites \\
\hline$\left\langle\nu_{1}\right\rangle\left(\mathrm{cm}^{-1}\right)$ & 28.66 & 16.71 & \multirow{2}{*}{\multicolumn{3}{|c|}{19.62}} \\
\hline$\Delta^{(1 / \lambda)}\left(\mathrm{cm}^{-1}\right)$ & 7.85 & 6.66 & & & \\
\hline$\tau_{c}(\mathrm{ps})$ & 0.0507 & 0.0584 & \multicolumn{3}{|c|}{$\cdots$} \\
\hline$\Delta \tau_{c}$ & 0.075 & 0.073 & \multicolumn{3}{|c|}{$\ldots$} \\
\hline $2 \Delta^{2} \tau_{c}$ & 1.18 & 0.99 & \multicolumn{3}{|c|}{$\cdots$} \\
\hline $\begin{array}{l}\nu_{1, \text { spectrum }}\left(\mathrm{cm}^{-1}\right) \text { with } t_{n}=2.5 \\
\quad \text { and } t_{m}=15(\mathrm{ps})\end{array}$ & 28.68 & 16.70 & \multicolumn{3}{|c|}{$\cdots$} \\
\hline $\begin{array}{l}\text { FWHM }_{\text {spectrum }}\left(\mathrm{cm}^{-1}\right) \text { with } t_{n}=2.5 \text {, } \\
\quad \text { and } t_{m}=15(\mathrm{ps})\end{array}$ & $1.25 \pm 0.03$ & $0.98 \pm 0.03$ & \multicolumn{3}{|c|}{$\cdots$} \\
\hline $\begin{array}{c}\nu_{1, \text { spectrum }}\left(\mathrm{cm}^{-1}\right) \text { with } t_{n}=2.0, \text { and } \\
t_{m}=50(\mathrm{ps})\end{array}$ & 28.66 & 16.71 & 28.67 & & 16.71 \\
\hline $\begin{array}{l}\mathrm{FWHM}_{\text {spectrum }}\left(\mathrm{cm}^{-1}\right) \text { with } t_{n}=2 \\
\quad \text { and } t_{m}=50(\mathrm{ps})\end{array}$ & 1.23 & 1.04 & 1.26 & & 1.06 \\
\hline
\end{tabular}




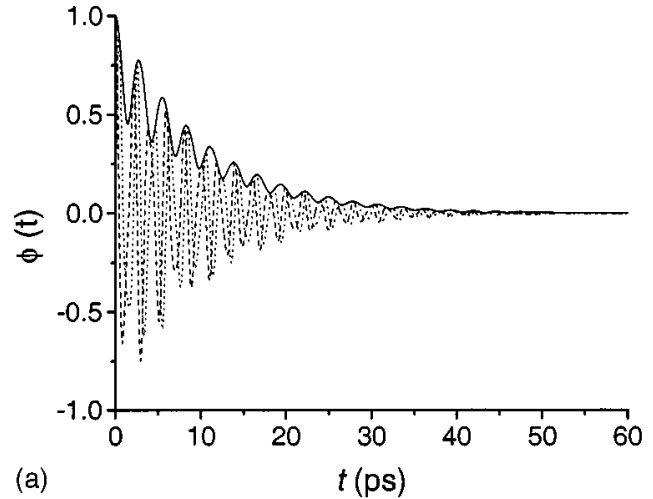

(a)

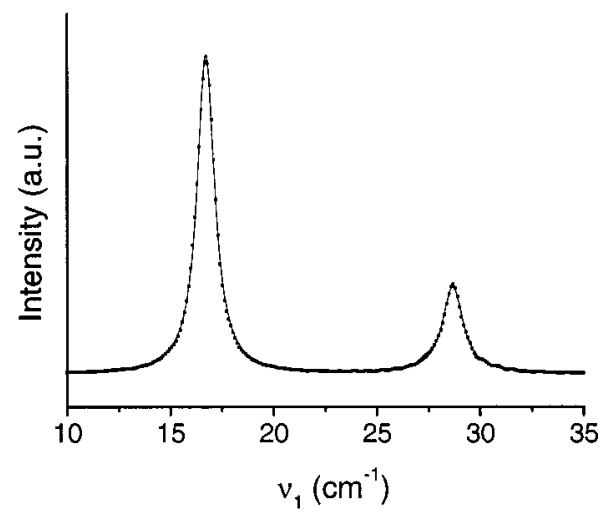

FIG. 6. (a) Relaxation function of vibrational frequency of nitrogen in the solid $\delta$ phase at $170 \mathrm{~K}$ and $7.0 \mathrm{GPa}$ obtained without distinguishing between the $a$ and $c$ sites; dashed line, real part; dotted line, imaginary part; solid line, absolute value. (b) Calculated spectrum (circles) of nitrogen at $170 \mathrm{~K}$ and $7.0 \mathrm{GPa}$ in the solid $\delta$ phase; Fourier transformation of the relaxation function given in (a). The line represents a Lorentzian fit of the calculated spectrum.

tral line shape of the molecules on the $a$ sites deviates from the Kubo predictions in the fast modulation regime, since the FWHM is larger than $2 \Delta^{2} \tau_{c}$.

To show the usefulness of the method, the vibrational spectrum of nitrogen in the $\delta$ phase has also been calculated without separating the frequencies of the two sites as well. The results are again presented in Table II. The calculated relaxation function using the parameters $t_{n}=2 \mathrm{ps}$ and $t_{m}$ $=50 \mathrm{ps}$ is pictured in Fig. 6(a). The absolute value of the total relaxation function clearly does not decay exponentially, but for large $t$ an exponential function is approached. Therefore, one must choose a large value for $t_{m}$. The calculated spectrum is presented in Fig. 6(b). The calculated spectrum clearly shows two distinct peaks, in contrast to the total momentary distribution function in which the two peaks cannot be distinguished. The integrated intensity of the low frequency peak is three times higher than that of the high frequency peak. The position of the two peaks is the same as found in the first method. The spectrum can be fitted much better with a double Lorentzian than a double Gaussian curve. It can be seen that the calculated spectrum of especially the high frequency peak ( $a$ sites), with the fast decay of the relaxation function, is not smooth, caused by the large value of $t_{m}$. For comparison, the vibrational spectra of the $a$ and $c$ sites have been calculated using the same parameters for $t_{n}(2 \mathrm{ps})$ and $t_{m}(50 \mathrm{ps})$ and treating the frequencies of both sites separately. Note that in order to obtain smooth spectra this value for $t_{m}$ is too large, since for both sites, $\phi(50 \mathrm{ps})<0.01$, which leads to numerical errors. As shown in Table II, this calculation gives the same results for the peak position as well as the FWHM within accuracy of the calculations. Summarizing, the total momentary frequency distribution of nitrogen in the $\delta$ phase shows only one broad peak, but if the vibrational spectrum is calculated by considering the relaxation function as a single function, clearly two peaks can be distinguished in the calculated spectrum.

Guissani et al..$^{30}$ pointed out that, in case there are two or more inequivalent lattice sites, there is not a one-to-one correspondence between spectral lines and sites, due to resonant transfer. In the case of oxygen this leads to a considerable effect on the calculated relative band intensities. We did not take into account this resonant transfer because in the case of nitrogen its contribution is probably small. Experiments show ${ }^{31}$ that the low frequency band is approximately three times as intense as the high frequency one. This corresponds to the ratio of the number of the two sites.

\section{CONCLUSIONS}

The vibrational line shapes and linewidths of nitrogen have been calculated from MD simulations by determining the Fourier transform of the relaxation function. The calculated linewidth has been compared with the results obtained by using the Kubo expression for the fast modulation regime. The relevant Kubo parameter, $\Delta \tau_{c}$, of the systems investigated in this work varies from 0.03 to 0.23 .

Four systems of nitrogen in the fluid phase, with large differences in correlation time and/or amplitude of modulation, have been studied. The calculated line shape of the system with the lowest value of $\Delta \tau_{c}$, is in perfect agreement with the predictions for the line shape in the fast modulation regime: a Lorentzian line shape with a FWHM given by $2 \Delta^{2} \tau_{c}$. For the system with the largest value of $\Delta \tau_{c}$ both the linewidth and the line shape deviate slightly, while for the other two systems only the peak positions show small deviations from the average frequency toward the peak position of the momentary distribution. The results show that the method used in the previous work is justified. The procedure described in this paper is also very useful for systems in the fast modulation regime but with a long correlation time (which is difficult to determine accurately), since this method does not use the value of $\tau_{c}$. For large $t$ (compared to $\tau_{c}$ ) the relaxation functions of the four systems investigated indeed decay exponentially, in agreement with the predictions of Kubo.

In the fast modulation regime the linewidth can also be expressed in terms of the dephasing time $T_{2}$, the exponent of the decay of the relaxation function. For the four systems investigated, this gives consistent results.

In addition, the vibrational spectrum of solid $\delta$ - $\mathrm{N}_{2}$ has been calculated. The present procedure reveals the differences in dynamical behavior of the molecules at the two different sites, which is reflected in the linewidth compared to $2 \Delta^{2} \tau_{c}$, although the Kubo parameter has the same value. Thus, this example shows that the Kubo parameter does not provide an unambiguous criterion for the fast modulation 
regime. It also shows that the correlation time, defined by the integration over the correlation function, does not provide sufficient information about the frequency autocorrelation. In the case of the $a$ sites the small value of the correlation time is misleading, since the frequency correlation has a finite value for much longer times.

If the spectrum of $\delta$ - $\mathrm{N}_{2}$ is calculated without separating the frequencies of the two sites, it is shown that the calculated vibrational spectrum consists of two distinct peaks, even though these peaks cannot be directly distinguished in the total momentary distribution function. The peak positions and linewidths are the same as those obtained by analyzing the two sites separately. Therefore, this method can also be applied if one does not know whether or not closely spaced lines are to be expected.

It has been shown that the present method for calculating vibrational spectra using the relaxation function works quite well for systems with a large variety in the value of the Kubo parameter and the relaxation time. Therefore, this method can also be used to calculate line shapes in the intermediate Kubo regime, where no other means are available. The calculations for these cases will be presented in paper II.

${ }^{1}$ R. Kubo, in Fluctuation, Relaxation and Resonance in Magnetic Systems, edited by D. Ter Haar (Oliver and Boyd, Edinburgh, 1962).

${ }^{2}$ S. F. Fischer and A. Laubereau, Chem. Phys. Lett. 35, 6 (1975).

${ }^{3}$ W. G. Rothschild, J. Chem. Phys. 65, 455 (1976); 65, 2958 (1976).

${ }^{4}$ R. M. Lynden-Bell, Mol. Phys. 33, 907 (1977).

${ }^{5}$ S. R. J. Brueck, Chem. Phys. Lett. 50, 516 (1977).

${ }^{6}$ D. W. Oxtoby, D. Levesque, and J. J. Weis, J. Chem. Phys. 68, 5528 (1978).

${ }^{7}$ D. Levesque, J. J. Weis, and D. W. Oxtoby, J. Chem. Phys. 79, 917 (1983).
${ }^{8}$ D. Levesque, J. J. Weis, and D. W. Oxtoby, J. Chem. Phys. 72, 2744 (1980).

${ }^{9}$ K. S. Schweizer and D. Chandler, J. Chem. Phys. 76, 2296 (1982).

${ }^{10}$ M. F. Herman and B. J. Berne, J. Chem. Phys. 78, 4103 (1983).

${ }^{11}$ J. Chesnoy and J. J. Weis, J. Chem. Phys. 84, 5378 (1986).

${ }^{12}$ R. M. Lynden-Bell and P. O. Westlund, Mol. Phys. 61, 1541 (1987).

${ }^{13}$ J. Belak, R. D. Etters, and R. LeSar, J. Chem. Phys. 89, 1625 (1988).

${ }^{14}$ A. B. Meyers and F. Markel, Chem. Phys. 149, 21 (1990).

${ }^{15}$ J. Chesnoy and G. M. Gale, Adv. Chem. Phys. 70, 299 (1988).

${ }^{16}$ G. S. Devendorf and D. Ben-Amotz, J. Phys. Chem. 97, 2307 (1993).

${ }^{17}$ J. P. J. Michels, M. I. M. Scheerboom, and J. A. Schouten, J. Chem. Phys. 103, 8338 (1995).

${ }^{18}$ G. S. Devendorf, D. Ben-Amotz, and L. E. S. de Souza, J. Chem. Phys. 104, 3479 (1996).

${ }^{19}$ J. P. J. Michels, M. I. M. Scheerboom, and J. A. Schouten, J. Chem. Phys. 105, 9748 (1996).

${ }^{20}$ J. P. J. Michels and J. A. Schouten, Mol. Phys. 91, 253 (1997).

${ }^{21}$ B. Khalil-Yahyavi, M. Chatelet, and B. Oksengorn, J. Chem. Phys. 89, 3573 (1988).

${ }^{22}$ B. Lavorel, R. Chaux, R. Saint-Loup, and H. Berger, Opt. Commun. 62, 25 (1987).

${ }^{23}$ N. Gayathri and B. Bagchi, J. Chem. Phys. 110, 539 (1999).

${ }^{24}$ R. D. Etters, V. Chandrasekharan, E. Uzan, and K. Kobashi, Phys. Rev. B 33, 8615 (1986).

${ }^{25}$ M. I. M. Scheerboom, J. P. J. Michels, and J. A. Schouten, J. Chem. Phys. 104, 9388 (1996).

${ }^{26}$ J. P. J. Michels, M. E. Kooi, and J. A. Schouten, J. Chem. Phys. 108, 2695 (1998).

${ }^{27}$ D. T. Cromer, R. L. Mills, D. Schiferl, and L. A. Schwalbe, Acta Crystallogr., Sect. B: Struct. Crystallogr. Cryst. Chem. 37, 8 (1981).

${ }^{28}$ R. L. Mills, B. Olinger, and D. T. Cromer, J. Chem. Phys. 84, 2837 (1986).

${ }^{29}$ H. Olijnyk, J. Chem. Phys. 93, 8968 (1990).

${ }^{30}$ Y. Guissani, D. Levesque, J. J. Weis, and D. W. Oxtoby, J. Chem. Phys. 77, 2153 (1982)

${ }^{31}$ T. Westerhoff, A. Wittig, and R. Feile, Phys. Rev. B 54, 14 (1996); T. Westerhoff and R. Feile, ibid. 54, 913 (1996). 